\title{
A FOUR-POINT BENDING TEST APPARATUS FOR MEASUREMENT- AND MODEL-BASED STRUCTURAL ANALYSIS
}

\author{
Cheng-Chieh WU ${ }^{1}$, Sven WEISBRICH ${ }^{2}$, Mathias BURGER ${ }^{2}$, Frank NEITZEL ${ }^{2}$ \\ 1 Bundesanstalt für Materialforschung und -prüfung (BAM), Unter den Eichen 87, 12205 Berlin, Ger- \\ many, E-mail: cheng-chieh.wu@bam.de; \\ 2 Technische Universität Berlin, Strasse des 17. Juni 135, 10623 Berlin, Germany
}

\section{Introduction}

By means of a small-scale truss bridge, the ability of the Measurement- and Model-based Structural Analysis to detect and localize damage was examined in [1]. Although there was no noteworthy difficulty in detecting damage, it turned out that damage localization responds sensitively to systematic influences, i.e. non-modelled properties of the mechanical model. Therefore, another experiment is being conducted to re-examine the Measurementand Model-based Structural Analysis. For this purpose, the bending test is carried out as it has been already theoretically respectively numerically discussed in [2]. In this attempt, the systematic influences such as residual stress are kept as low as possible.

\section{Specimen and experimental set-up}

The specimen is a $1.5 \mathrm{~m}$ long slender aluminium beam with a square cross-section of $35 \mathrm{~mm}$ by $35 \mathrm{~mm}$, see Fig. 1. The beam was designed with small indentations. They ensure that the applied and reactive forces always act in the same place on the beam specimen. On both ends of the lower side of the beam, there are indentations for the bearings. The notches are located $1 \mathrm{~cm}$ from the outer edge of

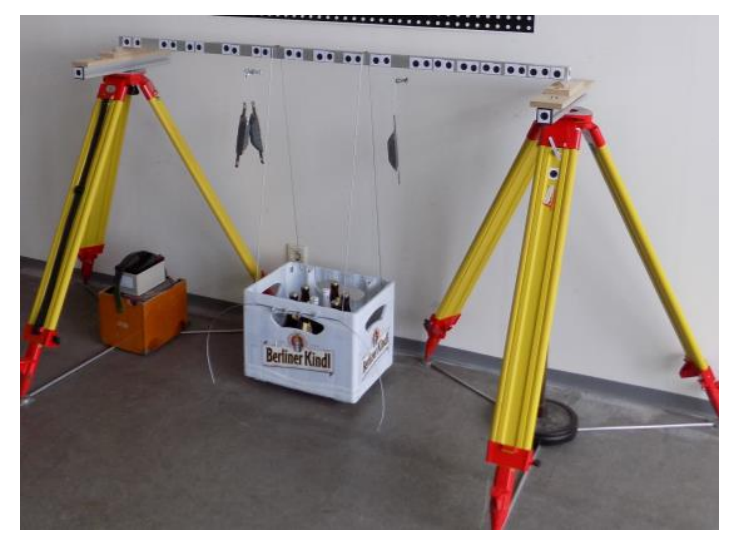

Fig. 1. A six-point bending test apparatus for an aluminium beam specimen. the beam. The bearings consist of a metal chamfer strip glued to a wooden structure. An aluminium profile was used to connect the bearing to the tripod. The tripod was placed on top of a metal star. To prevent the tripod from slipping, the tripod spider was glued to the floor with double-sided adhesive tape. In addition, weights were placed on the stand spider. On the upper side of the beam there are four indentations for attaching weights. Damage is caused by drilling and sawing the beam.

\section{Measurement system}

Photogrammetry is used to measure the deformation of the beam. To track the local displacements, in total 34 round target stickers are applied on the surface of the beam (31 markers) as well as on the tripod (three markers). The evaluation software has been developed by the Institute of Geodesy and Geoinformation Science at the Technische Universität Berlin to determine the position of the markers. Accordingly, the camera calibration and distortion corrections were carried out by them.

\section{Calibration of the reference state}

To adjust the elastic modulus of an undamaged slender beam, twelve experiments were conducted. In each experiment, the deformation behavior of the beam is examined in either unloaded or loaded state. For each beam state, images are taken at short intervals. The exposure time was also considered when determining the intervals. A total of 12 by $300 \mathrm{ob}-$ servations is obtained for each of the 31 markers.

The variance-covariance matrix of the marker position observations is determined by the measurements of the entire experiment. The standard deviation of the displacement in $y$-direction is

$$
\sigma_{u}=0.003 \mathrm{~mm}
$$

and the adjusted elastic modulus is

$$
\widehat{E}=67.397 \mathrm{GPa} \text { with } \sigma_{\hat{E}}=0.009 \mathrm{~mm} \text {. }
$$




\section{Damage detection and localization}

In the same way as in [2], the presented approach is followed to detect and localize damage. However, to avoid long computation time, in case where the global test failed to reject the null hypothesis, the standardized residuals $\mathrm{NV}_{\zeta}$ of the observed unknown elastic parameters are evaluated. The finite element discretization of the beam specimen is determined in dependence on the attached markers as well as the application points of the forces and bearings. Thus, the finite element model of the beam consists of 36 non-equidistant elements. Considering the two boundary conditions and a linear interpolation of the elastic parameter of each element, a total of 39 unknowns result. Due to the high degree of freedom resulting from the number of unknowns, an incorrect adjustment of the boundary conditions can occur. Eventually, the elastic parameters of the elements can be incorrectly adjusted to counteract the effects of yielding bearings. Therefore, in a first step, all elements share the same elastic modulus. In other words, one Young's modulus and two boundary conditions must be determined from the displacement observations. Then, in the second step, the adjusted boundary conditions are used as fixed values, while the 36 elastic parameters are determined from the displacement observations.

The beam was gradually damaged at a fixed position. The edge-to-edge length of the beam is $1500 \mathrm{~mm}$. The damage was caused at approximately $383 \mathrm{~mm}$, measured from the right edge. The beam length in the finite element model is $1480 \mathrm{~mm}$ which corresponds to the distance between the bearings. Thus, the damage position is at approximate $1107 \mathrm{~mm}$. The damage has been successively increased. First, the beam was drilled through with a radius of $4 \mathrm{~mm}$. Six different load experiments were then carried out. The damage was not detected in five out of six cases. And the localization of the fault failed where an alleged damage was detected. The beam was then further damaged. The borehole was extended to $10 \mathrm{~mm}$ radius; then two more holes were drilled with $10 \mathrm{~mm}$ radius each, and damage was further increased. Again, no damage was noticeably detected. Then, the beam was sawed. Here, it was observed that if the attached weights were large enough, the damage was detected but the localization of the damage failed. Ultimately, the damage was large enough, so that the damage could be detected and localized repeatedly. The damage position is at approximate $1107 \mathrm{~mm}$. Thus, it affects the element node $\zeta=24$ which is at $1112 \mathrm{~mm}$. How- ever, according to the performed analysis, the damage is located at the element node $\zeta=22$ which is at $990 \mathrm{~mm}$. This results in an error estimate of $117 \mathrm{~mm}$. In relation to the total length of $1480 \mathrm{~mm}$, the mislocalization is less than 8 percent, $(117 \mathrm{~mm}$ $/ 1480 \mathrm{~mm} \approx 0.079$ ).

\section{Conclusion}

By means of a beam bending experiment, the reevaluation has shown that the Measurement- and Model-based Structural Analysis can detect and localize damage. However, the likelihood of localizing damage is hampered by systematic influences. Here, in this case, it was observed that ambient light affected the photogrammetric system. Ambient light changes, for example, due to the influence of clouds. As a result, the pixels on the images change their contrasts and thus influencing the adjusting circular position of the marker. It is also inevitable that the markers will become soiled over time. This also impacts an apparent change in the marker position. Subsiding tripods and bearings were also unhelpful in reducing systematic influences during evaluation. The maximum deflection was approximately $1.4 \mathrm{~mm}$ and due to the subsiding of roughly $0.1 \mathrm{~mm}$, the elastic parameter was missing $3 \mathrm{GPa}$ at the end of the adjustment. To counteract the subsidence, on the one hand the finite element model had to be extended, on the other hand the attached weight should not become too large. Since the beam was very stiff and it was not possible to attach too much weight, the deflection became too small. But it was necessary that the deflection had to be large enough to overcome the noise and systematic influences of photogrammetry. In the end, there was no other choice but to increase the damage to the beam. This made it possible to achieve consistent damage detection and localization.

\section{References}

[1] Wu, C.-C., Kadoke, D., Fischer, M., Kohlhoff, H., Weisbrich, S., Neitzel, F., A small-scale test bridge for Measurement- and Model-based Structural Analysis, In 35th Danubia-Adria Symposium on advances in experimental mechanics, Sinaia, 25-28 Sep, 2018, PRINTECH, Bucureşti, 2018, pp. 23-24.

[2] Wu, C.-C., Weisbrich, S., Neitzel, F., Integrated structural analysis of hybrid measurement and finite element method for damage detection within a slender beam. In 31st Danubia-Adria Symposium on advances in experimental mechanics, Kempten, 24-27 Sep, 2014, VDI Verein Deutscher Ingenieure e.V., Düsseldorf, 2014, pp. 191-192. 